\title{
Pedogenesis of Volcanic Ash Soils in Andean Ecuador
}

\author{
F. Zehetner, W. P. Miller,* and L. T. West
}

\begin{abstract}
Weathering and development of volcanic ash soils show similar patterns in different regions of the world; however, the specific environmental conditions at a given location result in a unique combination of factors and processes governing soil formation. This research was conducted to study pedogenesis on volcanic slopes in the interAndean valley of northern Ecuador. Twelve pedons representing different pedogenic environments were sampled at elevations between 2410 and $4050 \mathrm{~m}$ above sea level (asl). In pedons above $3200 \mathrm{~m}$ asl, allophane and $\mathrm{Al}$-humus complexes dominate the colloidal fraction, the topsoils are high in organic matter, and the soils classify as Andisols with melanic epipedons. In pedons below $2700 \mathrm{~m}$ asl, halloysite is the predominant colloidal constituent, the topsoils contain $<1 \%$ organic $C$, and the soils are Inceptisols and Entisols. The pedons at intermediate elevations mark a transition zone, in which allophane and halloysite coexist and the soils generally classify as Andisols with umbric epipedons. This general altitudinal pattern was found to be altered in unstable landscape positions, where andic soil properties have been removed along with the erosion of topsoil material. Virtually the same altitudinal weathering sequence was observed in the 3000-yr-old soils and in buried paleosols, which are considered to be older than 40000 years. Thus, different time of pedogenesis has not caused marked differences in the composition of the colloidal fraction. Climate is considered the overriding factor responsible for the observed altitudinal differences in soil development by affecting leaching regime and organic matter decomposition.
\end{abstract}

$S^{\circ}$ ILS DERIVED FRom volcanic deposits exhibit unique physical and chemical properties, such as low bulk density, high water retention, variable charge characteristics, and strong phosphate sorption, which have been largely ascribed to active amorphous weathering products, such as allophane, imogolite, and Al-humus complexes (e.g., Shoji et al., 1993; Kimble et al., 2000). The extent to which these properties are expressed in a volcanic ash soil depends therefore on its stage of soil development. Various weathering sequences have been proposed to explain the formation and transformation of colloidal constituents in volcanic ash soils under different environmental conditions.

The formation of Al-humus complexes has been observed in recent and buried A horizons of volcanic ash soils, and has been reported to be favored by conditions unfavorable to the formation of allophane (Shoji et al., 1982; Shoji and Fujiwara, 1984). Aluminum-humus complexes are predominant at $\mathrm{pH}\left(\mathrm{H}_{2} \mathrm{O}\right)<5$, whereas the precipitation of allophane is favored above $\mathrm{pH}$ 5. It has been further suggested that the precipitation of allophane is impeded by competition between humic sub-

F. Zehetner, W.P. Miller, and L.T. West, Dep. of Crop and Soil Sciences, Univ. of Georgia, Athens, GA 30602. Received 30 Sept. 2002.*Corresponding author (wmiller@uga.edu).

Published in Soil Sci. Soc. Am. J. 67:1797-1809 (2003).

(c) Soil Science Society of America

677 S. Segoe Rd., Madison, WI 53711 USA stances and orthosilicic acid for soluble Al (Wada, 1989; Shoji et al., 1993).

Allophane and halloysite are the dominant components in the clay fraction of many volcanic ash soils around the world. It has been suggested that halloysite forms over time upon weathering of allophane (e.g., Wada, 1989); however, many studies have revealed that volcanic ash may weather directly to halloysite as well as to allophane (e.g., Parfitt et al., 1983; Parfitt et al., 1984; Parfitt and Wilson, 1985; Singleton et al., 1989). Silicon activity in the soil solution has been suggested as the overriding factor responsible for the preferential formation of either halloysite or allophane. Parfitt and Wilson (1985) and Singleton et al. (1989) found that halloysite was predominant when $\mathrm{H}_{4} \mathrm{SiO}_{4}^{0}$ in soil solution exceeded 250 and $350 \mu \mathrm{mol} \mathrm{L}{ }^{-1}$, respectively, and allophane predominated below these concentrations.

A variety of factors can control soil solution chemistry and hence affect the mineralogy of a soil's colloidal fraction. Wada (1987) has suggested that in contrast with allophane, the formation of halloysite is favored by a thick depositional overburden that produces a silica-rich environment. Singleton et al. (1989) and Bakker et al. (1996) reported impeded drainage to favor halloysite formation, whereas the presence of allophane was associated with well-drained soil horizons. Parfitt et al. (1983) proposed a weathering scheme for rhyolitic ash, in which halloysite is the dominant clay mineral where the mean annual precipitation is less than approximately $1500 \mathrm{~mm}$, and allophane predominates above $1500 \mathrm{~mm}$. Along these lines, many subsequent studies on volcanic ash soils in different parts of the world have confirmed the importance of rainfall and leaching in mineral formation (e.g., Parfitt et al., 1984; Stevens and Vucetich, 1985; Takahashi et al., 1993; Nizeyimana et al., 1997; Nieuwenhuyse et al., 2000).

Toposequences of soils along the slopes of volcanoes have been studied to assess the effect of climate on weathering and mineral formation (Chartres and Pain, 1984; Nizeyimana et al., 1997). On an extinct volcano in Rwanda, Nizeyimana et al. (1997) found decreasing allophane contents and increasing halloysite contents with decreasing elevation, which they attributed to a concomitant decrease in rainfall. Chartres and Pain (1984) found a similar weathering pattern with elevation in highland Papua New Guinea, although there precipitation increased with decreasing elevation. They suggested that lower rates of evapotranspiration with increasing altitude caused greater leaching, thus favoring the formation of allophane.

In addition to allophane and halloysite, 2:1-type phyl-

Abbreviations: $\mathrm{Al}_{\mathrm{p}}$, pyrophosphate-extractable $\mathrm{Al}$; asl, above sea level; BP, before present; DSC, differential scanning calorimetry; EG, ethylene glycol; ET, evapotranspiration; IR, infrared spectroscopy; subscript [o], acid oxalate-extractable; XRD, X-ray diffraction. 
losilicates with and without hydroxy-Al interlayering are commonly found in volcanic ash soils. Their occurrence has been ascribed to in situ pedogenic transformations (Shoji et al., 1982; Yamada and Shoji, 1983), eolian addition (Mizota and Takahashi, 1982; Mizota, 1983), incorporation into volcanic ash during eruption (Dudas and Harward, 1975; Pevear et al., 1982; Ping et al., 1988), and mixing of recent volcanic ash with underlying paleosols (Dudas and Harward, 1975).

The above discussion suggests that similar trends in weathering and development of volcanic ash soils exist in different parts of the world. However, specific environmental conditions, such as the eruption histories of surrounding volcanoes, the composition of volcanic ejecta, present-day climate, as well as paleoclimate and glaciation history, result in a unique combination of factors and processes governing soil formation at a given location. The pedogenesis of volcanic ash soils has been extensively studied in Japan and New Zealand; however, very little is known about weathering and soil formation on the slopes of volcanic peaks in the Andes. The objective of this study was, therefore, to investigate the factors and processes that affect soil development on volcanic slopes in the unique inter-Andean valley of northern Ecuador.

\section{MATERIALS AND METHODS}

\section{Study Area}

The study area is located on the slopes of volcano Cotacachi, about $35 \mathrm{~km}$ north of the equator (Fig. 1). The region has been largely deforested and the present landscape is dominated by agricultural land use below $3000 \mathrm{~m}$ asl, and high-altitude scrubland and grassland (matorral and páramo, respectively) above $3000 \mathrm{~m}$ asl. The climate in the area is that of an equatorial high-altitude environment, with temperatures almost constant throughout the year, but showing pronounced diurnal oscillations. The mean annual temperature is about $15^{\circ} \mathrm{C}$ in the town of Cotacachi, and drops by about $0.6^{\circ} \mathrm{C}$ per $100 \mathrm{~m}$ of elevation increase. The mean annual precipitation is about $900 \mathrm{~mm}$ at $2500 \mathrm{~m}$ asl and increases with elevation to about $1500 \mathrm{~mm}$ at $4000 \mathrm{~m}$ asl (Nouvelot et al., 1995). The annual rainfall distribution is characterized by an expressed seasonality with a dry season from June to August, and about $90 \%$ of the annual precipitation occurring from September to May.

The volcanic complex of Cotacachi has a long history of volcanic activity involving several different eruption centers,

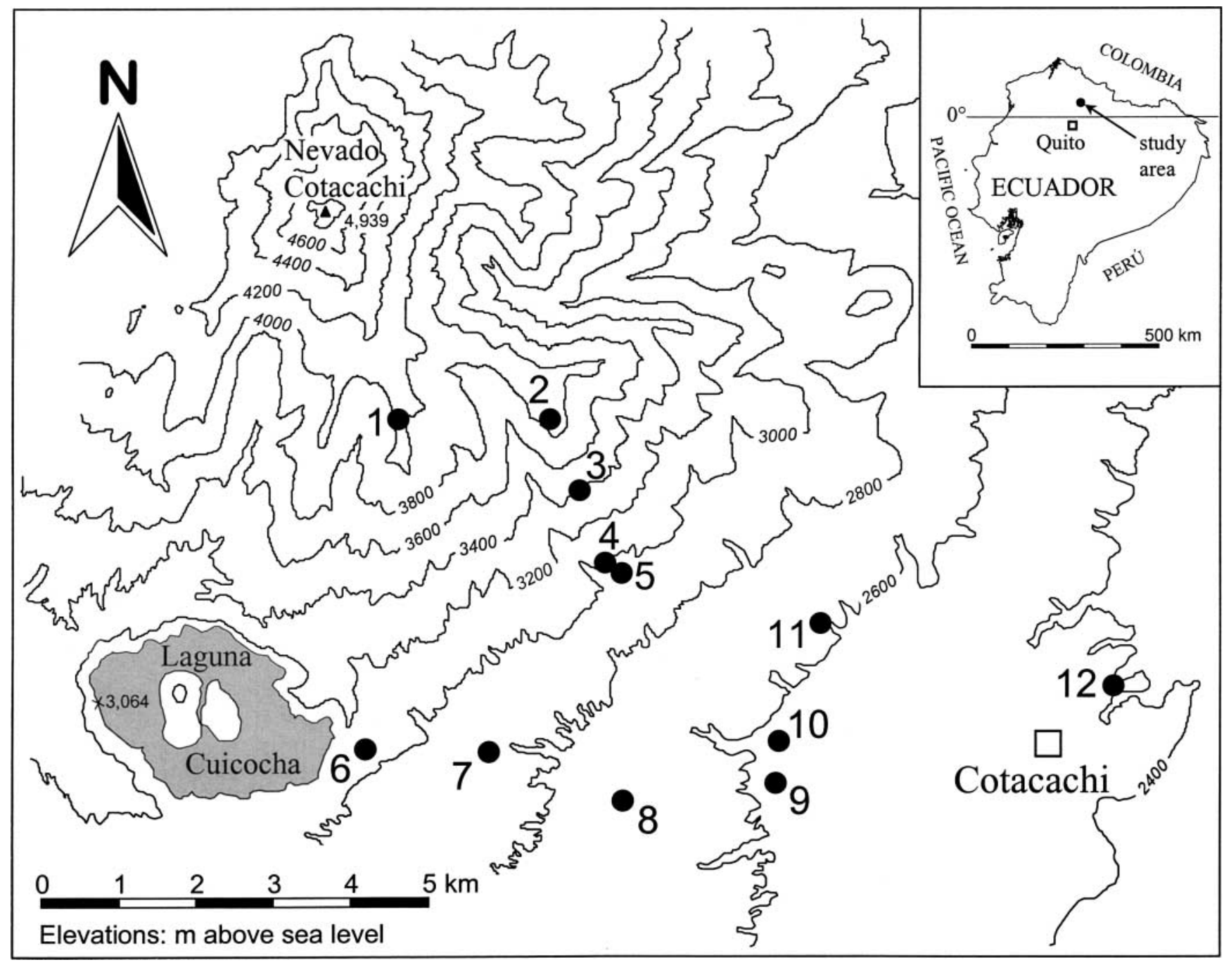

Fig. 1. Study area; the locations of the studied pedons are marked with solid dots, numbered 1 through 12. 
of which only Cuicocha has been active in the Holocene. The other centers have not erupted in the past $40000 \mathrm{yr}$ (Hall and Mothes, 1994). Volcano Cuicocha has had three phases of activity, which occurred over a period of a few hundred years, ending about 3000 yr BP (Mothes and Hall, 1991; Hall and Mothes, 1994; Athens, 1999). The present caldera of Cuicocha was formed by explosive eruptions that resulted in massive pyroclastic flows, which reach from Laguna Cuicocha past the town of Cotacachi (Fig. 1). Tephra falls from these eruptions cover the southern slopes of volcano Cotacachi. The soils under study have formed on these deposits, which have andesitic to dacitic composition. Pedons 1 through 5, 10, and 11 are located on tephra deposits, and Pedons 6 through 9 and 12 are located on pyroclastic flow deposits (Fig. 1). Selected site characteristics for the studied pedons are presented in Table 1. Small amounts (few centimeters) of tephra from later eruptions of surrounding volcanoes, notably Pululahua (2300 yr BP) and Quilotoa (800 yr BP), may have been deposited in the area (Hall and Mothes, 1994; Athens, 1999; Mothes and Hall, 1999) and mixed with preexisting soils.

\section{Methods}

Soils were described and sampled according to genetic horizons, air-dried, and passed through a $2-\mathrm{mm}$ sieve. Particlesize distribution was determined with a combined sieve and pipette method after removal of organic matter with hydrogen peroxide and dispersion with sodium metaphosphate (Soil Survey Staff, 1996). Total C was determined by dry combustion (Tabatabai and Bremner, 1991). The values thus obtained were assumed to correspond to organic $\mathrm{C}$, since the studied soils did not contain carbonate minerals. Water retention was measured after equilibration of rewet soil samples at $1.5 \mathrm{MPa}$ in a pressure-plate extractor, and clay content (\%) was estimated by $\left(\% \mathrm{H}_{2} \mathrm{O}\right.$ retained at $1.5 \mathrm{MPa}-\%$ organic $\left.\mathrm{C}\right) \times 2.5$ (Soil Survey Staff, 1998). Soil pH was measured in $\mathrm{H}_{2} \mathrm{O}$ and $1 \mathrm{M} \mathrm{KCl}$ at a soil/solution ratio of 1:2.5 after $30 \mathrm{~min}$ of equilibration and in $1 M \mathrm{NaF}$ at 1:50 after exactly 2 min (Soil Survey Staff, 1996). Phosphate retention was determined by measuring the percentage of $\mathrm{P}$ retained from $25 \mathrm{~mL}$ of a solution containing $1000 \mathrm{mg} \mathrm{L}^{-1}$ of $\mathrm{P}$ and buffered at $\mathrm{pH} 4.6$ after shaking with $5 \mathrm{~g}$ of soil for $24 \mathrm{~h}$ (Soil Survey Staff, 1996).

Dissolution analyses were conducted according to Wada (1989). Aluminum associated with soil organic matter, $\mathrm{Al}_{\mathrm{p}}$, was estimated by extraction with $0.1 M$ sodium pyrophosphate at $\mathrm{pH} 10$ using a soil/solution ratio of 1:100 and shaking for $16 \mathrm{~h}$. Aluminum and silicon associated with active amorphous constituents, $\mathrm{Al}_{\mathrm{o}}$ and $\mathrm{Si}_{\mathrm{o}}$, respectively, were estimated by ex- traction of ground $(<0.25 \mathrm{~mm}$ ) samples with $0.175 \mathrm{M}$ ammonium oxalate-oxalic acid at $\mathrm{pH} 3$ using a soil/solution ratio of 1:100 and shaking for $4 \mathrm{~h}$ in the dark. Aluminum and silicon were measured by inductively coupled plasma-mass spectrometry (ICP-MS). The molar Al/Si ratio of allophane was calculated from $\left(\mathrm{Al}_{\mathrm{o}}-\mathrm{Al}_{\mathrm{p}}\right) / \mathrm{Si}_{\mathrm{o}}$, and the amount of allophane in the samples was estimated by multiplying $\mathrm{Si}_{\mathrm{o}}$ by $5,6,7$, or 10 if the $\mathrm{Al} / \mathrm{Si}$ ratio was approximately $1,1.5,2$, or 2.5 , respectively (Parfitt, 1990).

For mineralogical analyses, the clay fraction $(<2 \mu \mathrm{m})$ was separated from the soil samples by repeated dispersion and centrifugation after initial dispersion with ultrasonic vibration (Hunter and Busacca, 1989). The clay samples were analyzed with differential scanning calorimetry (DSC) at temperatures between 35 and $600^{\circ} \mathrm{C}$ and infrared (IR) spectroscopy at wavenumbers between 400 and $4000 \mathrm{~cm}^{-1}$. Oriented clay samples were prepared for X-ray diffraction (XRD) analysis using the filter-membrane technique (Drever, 1973) with the following saturation treatments: Mg saturation, air-dried, and ethylene glycol solvated; K saturation, air-dried, and heated to 110 , 300 , and $550^{\circ} \mathrm{C}$. The samples were scanned from 3 to $32^{\circ} 2 \theta$ with $\mathrm{CuK} \alpha_{1,2}$ radiation and a curved crystal monochromator.

\section{RESULTS General Soil Properties}

Selected characteristics of the studied pedons are presented in Table 2. In eight of the twelve pedons, the recent soil is overlying a paleosol formed on older volcanic deposits, and in three pedons $(9,10$, and 11$)$, more than one volcanic paleosol is found within a depth of $300 \mathrm{~cm}$

Formed on sandy and silty volcanic deposits, the studied soils generally exhibit low clay contents; however, in high-elevation pedons, incomplete dispersion of soil samples may have led to an underestimation of clay contents measured with the pipette method, as indicated by considerably lower values than estimated from $\mathrm{H}_{2} \mathrm{O}$ retention (Table 2). Topsoil clay contents increase with elevation, and in pedons above $2700 \mathrm{~m}$ asl, they are consistently higher than in underlying recent $\mathrm{Bw}$ and $\mathrm{C}$ horizons. On the other hand, clay contents of recent A horizons do not differ greatly from those of underlying recent $\mathrm{Bw}$ and $\mathrm{C}$ horizons in pedons below $2700 \mathrm{~m}$ asl.

Table 1. Site information for the studied pedons.

\begin{tabular}{|c|c|c|c|c|c|c|}
\hline Pedon & Location & Northing $\dagger$ & Easting $\dagger$ & Elevation & Land use & Soil classification $\div$ \\
\hline & & & 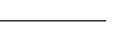 & m asl§ & & \\
\hline 1 & Antenas & 37602 & 796351 & 4050 & PáramoI & Vitric Melanocryands \\
\hline 2 & Cerro Huanansí & 37596 & 798308 & 3900 & Páramo & Vitric Melanocryands \\
\hline$\overline{3}$ & Cerro Huanansí & 36668 & 798689 & 3400 & Matorral\# & Pachic Vitric Melanudands \\
\hline 4 & Cerro Huanansí & 35731 & 799010 & 3000 & Pasture & Andic Dystrudepts \\
\hline 5 & Iltaquí Alto & 35600 & 799229 & 2950 & Cropland & Humic Údivitrands \\
\hline 6 & La Piyaba & 33341 & 795921 & 3060 & Matorral & Humic Udivitrands \\
\hline 7 & Morochos & 33293 & 797508 & 2900 & Cropland & Humic Udivitrands \\
\hline 8 & Morochos & 32664 & 799252 & 2740 & Cropland & Humic Udivitrands \\
\hline 9 & Chilcapamba & 32899 & 801233 & 2570 & Cropland & Vitrandic Dystrudepts \\
\hline 10 & Iltaquí Bajo & 33429 & 801258 & 2560 & Cropland & Vitrandic Udorthents \\
\hline 11 & Topo Grande & 34939 & 801793 & 2630 & Cropland & Vitrandic Udorthents \\
\hline 12 & Pilchibuela & 34146 & 805607 & 2410 & Forest & Vitrandic Udorthents \\
\hline
\end{tabular}

$\dagger$ Datum: Provisional South American 1956; Ellipsoid: International; Projection: UTM; Zone: 17N.

+ According to Soil Survey Staff (1998).

$\S$ asl, above sea level.

II Local term for high-altitude grassland.

\# Local term for scrubland. 
Table 2. Selected characteristics of the studied pedons.

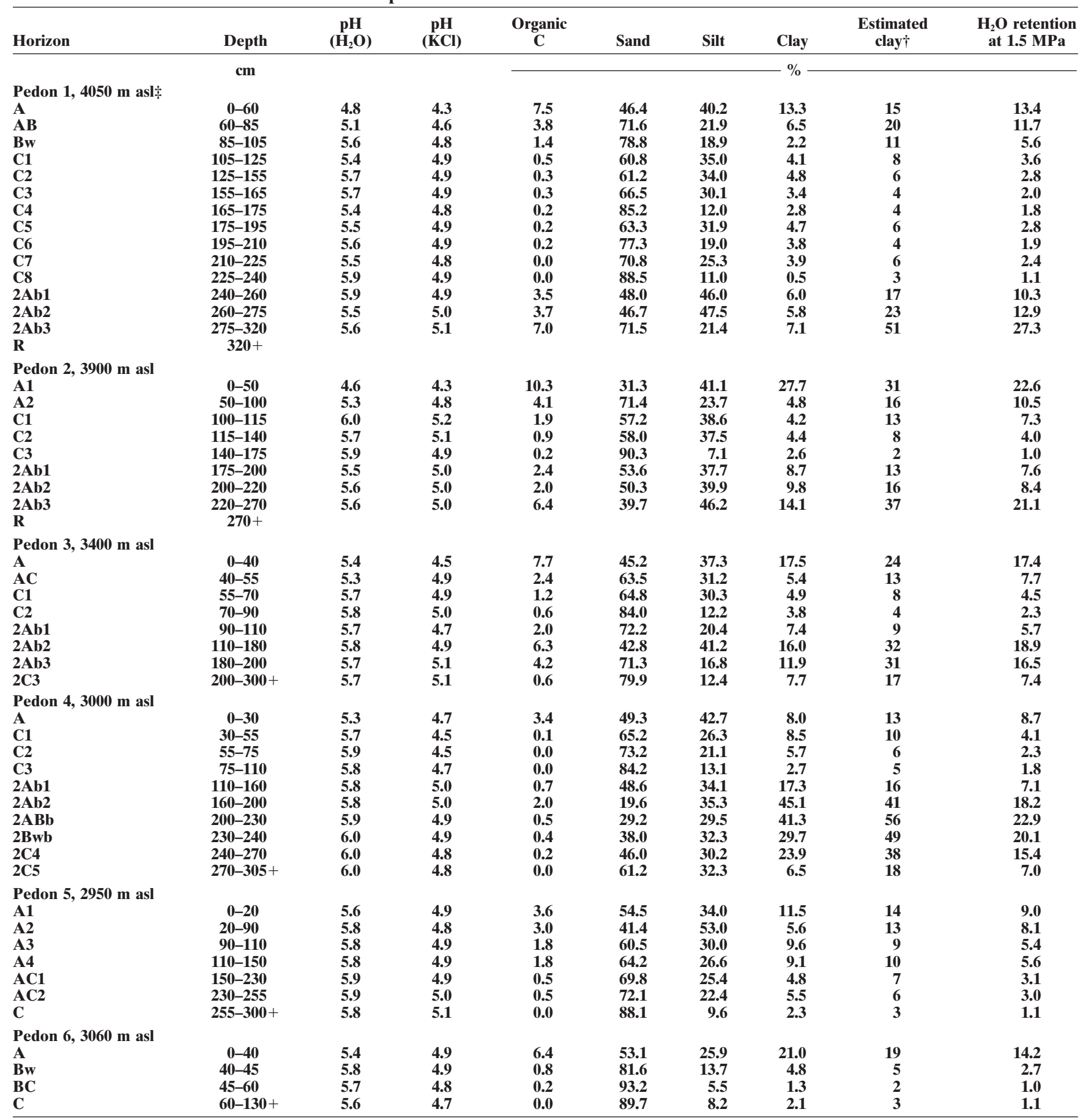

Continued next page.

The clay contents of paleosols are generally higher than those of recent soils.

Organic C contents of both recent and buried A horizons increase with altitude. Given the same age of the recent parent materials, average $\mathrm{C}$ sequestration rates appear to be an order of magnitude higher in high-elevation pedons compared with low-elevation pedons. Soil $\mathrm{pH}\left(\mathrm{H}_{2} \mathrm{O}\right)$ values decrease with increasing elevation, and are generally lower in recent $\mathrm{A}$ horizons than in underlying soil horizons including buried A horizons.
In all the studied pedons, $\mathrm{pH}(\mathrm{KCl})$ is consistently lower than $\mathrm{pH}\left(\mathrm{H}_{2} \mathrm{O}\right)$, indicating exchangeable acidity in these soils.

\section{Amorphous Colloidal Constituents}

The results of the dissolution analyses and properties related to amorphous material are presented in Table 3. In pedons at elevations above $3200 \mathrm{~m}$ asl, $\mathrm{pH}(\mathrm{NaF})$ values are generally above 9.4 in the entire profile, that 
Table 2. Continued.

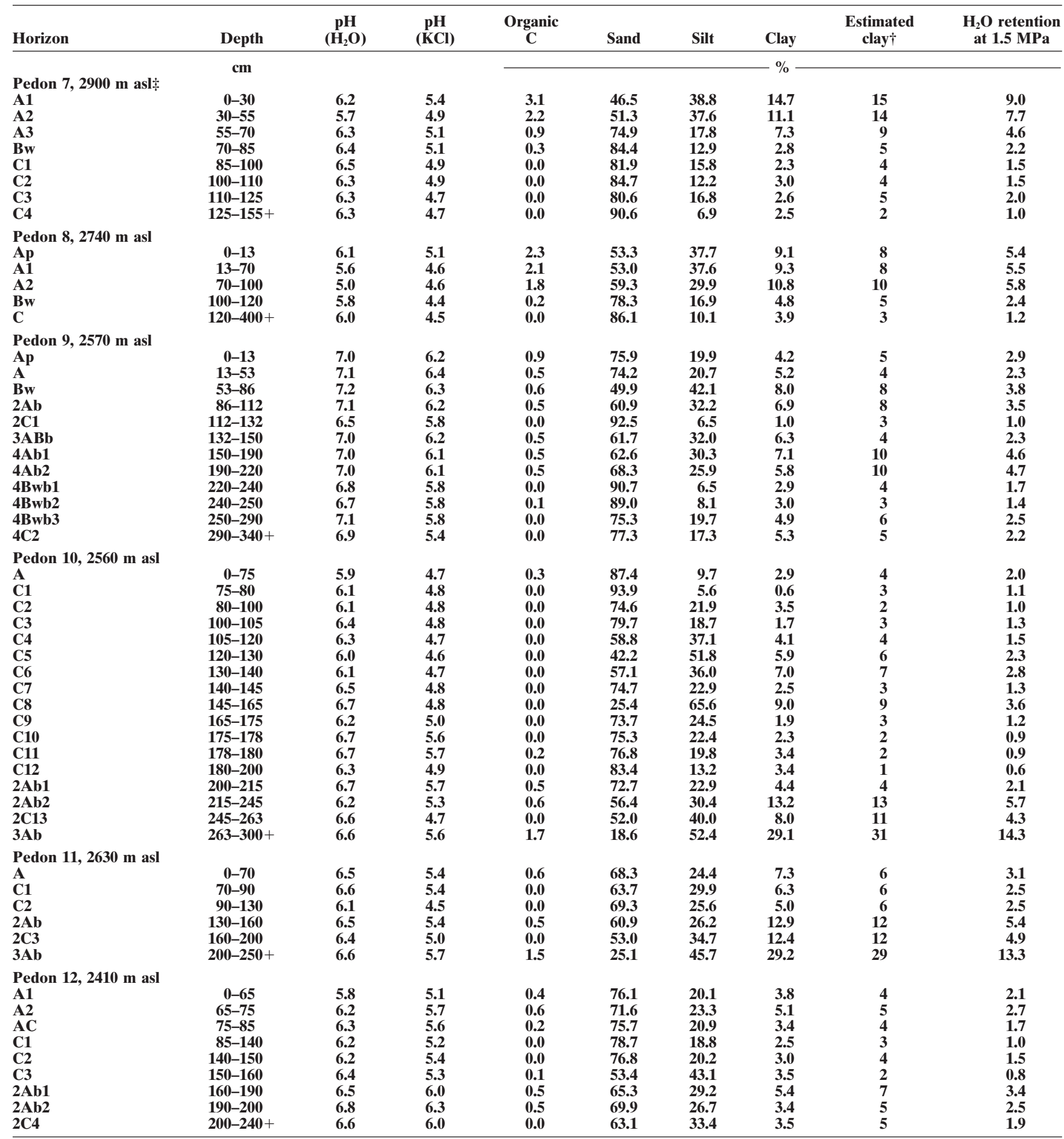

$\dagger$ Estimated clay $(\%)=\left(\% \mathbf{H}_{2} \mathrm{O}\right.$ retained at $1.5 \mathrm{MPa}-\%$ organic C $) \times 2.5($ Soil Survey Staff, 1998$)$.

$\uparrow$ asl, above sea level.

is, in recent soils and underlying paleosols. This indicates the dominant presence of active $\mathrm{Al}-\mathrm{OH}$ groups in the exchange complex of these soils (Wada, 1980). In pedons between 3200 and $2700 \mathrm{~m}$ asl, $\mathrm{pH}(\mathrm{NaF})$ values are above 9.4 only in the upper horizons, and in pedons below $2700 \mathrm{~m}$ asl, $\mathrm{pH}(\mathrm{NaF})$ values are below 9.4 in all horizons. Phosphate retention shows similar trends as $\mathrm{pH}(\mathrm{NaF})$ with the highest values in high-elevation ped- ons, high values in the upper horizons of pedons at intermediate elevations, and low values in all horizons of low-elevation pedons.

Pyrophosphate-extractable aluminum $\left(\mathrm{Al}_{\mathrm{p}}\right)$ is correlated with organic $\mathrm{C}(r=0.91 * * *)$ and generally increases with elevation. Acid oxalate-extractable aluminum and silicon $\left(\mathrm{Al}_{0}\right.$ and $\mathrm{Si}_{0}$, respectively) confirm the trends observed for $\mathrm{pH}(\mathrm{NaF})$ and $\mathrm{PO}_{4}$ retention, and 
Table 3. Dissolution analyses and properties related to amorphous material in the studied pedons.

\begin{tabular}{|c|c|c|c|c|c|c|c|c|c|c|}
\hline Horizon & $\mathbf{A} \mathbf{l}_{p} \dagger$ & $\mathbf{A l}_{0} \%$ & $\mathbf{S i}_{0} \ddagger$ & $\mathbf{A} \mathbf{l}_{\mathrm{o}}-\mathbf{A} \mathbf{l}_{\mathrm{p}}$ & $\left(\mathbf{A} \mathbf{l}_{0}-\mathbf{A} \mathbf{l}_{\mathrm{p}}\right) / \mathbf{S \mathbf { i } _ { 0 }}$ & $\mathbf{A} \mathbf{l}_{\mathrm{p}} / \mathbf{A l}_{\mathbf{0}}$ & $\mathbf{A l _ { p }} / \mathbf{C}$ & Allophane & pH (NaF) & $\mathrm{PO}_{4}$ retention \\
\hline & - & 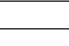 & $\mathbf{k g}^{-1}$ & - & -1 & $\operatorname{lar}$ & - & $\%$ & & $\%$ \\
\hline \multicolumn{11}{|l|}{ Pedon $1,4050 \mathrm{~m}$ asl $§$} \\
\hline & 7.0 & 12.0 & 3.9 & 5.0 & 1.4 & 0.6 & 0.04 & 2.3 & 10.82 & 90 \\
\hline AB & 4.4 & 15.6 & 6.6 & 11.2 & 1.8 & 0.3 & - & 4.6 & 10.48 & 89 \\
\hline Bw & 2.4 & 12.9 & 6.1 & 10.5 & 1.8 & 0.2 & - & 4.3 & 10.48 & 67 \\
\hline C1 & 0.7 & 7.7 & 4.1 & 7.0 & 1.8 & 0.1 & - & 2.9 & 10.47 & 46 \\
\hline C2 & 0.5 & 7.1 & 4.1 & 6.7 & 1.7 & 0.1 & - & 2.5 & 10.22 & 30 \\
\hline $\mathrm{C3}$ & 0.4 & 4.9 & 2.8 & 4.6 & 1.7 & 0.1 & - & 1.7 & 10.09 & 39 \\
\hline C4 & 0.2 & 4.2 & 2.4 & 3.9 & 1.7 & 0.1 & - & 1.4 & 9.58 & 23 \\
\hline C5 & 0.4 & 6.4 & 3.6 & 6.0 & 1.8 & 0.1 & - & 2.5 & 10.25 & 37 \\
\hline C6 & 0.2 & 4.2 & 2.5 & 4.0 & 1.7 & 0.1 & - & 1.5 & 9.79 & 24 \\
\hline C7 & 0.2 & 4.5 & 2.6 & 4.3 & 1.7 & 0.0 & - & 1.6 & 9.53 & 29 \\
\hline C8 & 0.1 & 2.6 & 1.6 & 2.5 & - & - & - & $<1$ & 9.35 & 14 \\
\hline $2 \mathrm{Ab} 1$ & 2.5 & 10.6 & 5.8 & 8.2 & 1.5 & 0.2 & 0.03 & 3.5 & 9.79 & 59 \\
\hline $2 \mathrm{Ab2}$ & 3.0 & 18.9 & 11.4 & 15.9 & 1.5 & 0.2 & 0.04 & 6.8 & 9.78 & 78 \\
\hline $2 \mathrm{Ab3}$ & 4.7 & 48.0 & 28.7 & 43.3 & 1.6 & 0.1 & 0.03 & 17.2 & 10.14 & 98 \\
\hline \multicolumn{10}{|l|}{ Pedon $2,3900 \mathrm{~m}$ asl } & 91 \\
\hline $\mathbf{A 2}$ & 4.9 & 15.5 & 6.5 & 10.6 & 1.7 & 0.3 & 0.05 & 3.9 & 10.96 & 87 \\
\hline C1 & 2.6 & 15.9 & 8.5 & 13.3 & 1.6 & 0.2 & - & 5.1 & 10.57 & 78 \\
\hline C2 & 1.5 & 9.4 & 5.5 & 7.9 & 1.5 & 0.2 & - & 3.3 & 10.26 & 52 \\
\hline $\mathbf{C 3}$ & 0.5 & 3.0 & 2.0 & 2.5 & 1.3 & 0.2 & - & 1.2 & 9.21 & 18 \\
\hline $2 \mathrm{Ab} 1$ & 2.9 & 10.2 & 5.1 & 7.3 & 1.5 & 0.3 & 0.05 & 3.1 & 10.16 & 65 \\
\hline $2 \mathrm{Ab2}$ & 2.5 & 10.5 & 5.6 & 8.0 & 1.5 & 0.2 & 0.05 & 3.3 & 10.03 & 67 \\
\hline $2 \mathrm{Ab3}$ & 7.8 & 22.9 & 11.6 & 15.1 & 1.4 & 0.3 & 0.05 & 6.9 & 9.82 & 92 \\
\hline \multicolumn{11}{|l|}{ Pedon $3,3400 \mathrm{~m}$ asl } \\
\hline AC & 4.0 & 15.8 & 7.1 & 11.8 & 1.7 & 0.3 & - & 4.3 & $\begin{array}{l}10.51 \\
10.82\end{array}$ & $\begin{array}{l}88 \\
79\end{array}$ \\
\hline C1 & 2.3 & 11.6 & 5.7 & 9.3 & 1.7 & 0.2 & - & 3.4 & 10.63 & 57 \\
\hline C2 & 1.1 & 5.8 & 3.1 & 4.7 & 1.6 & 0.2 & - & 1.9 & 9.90 & 30 \\
\hline $2 \mathrm{Ab} 1$ & 2.8 & 6.1 & 2.5 & 3.4 & 1.4 & 0.4 & 0.06 & 1.5 & 9.83 & 50 \\
\hline $2 \mathrm{Ab2}$ & 7.4 & 13.9 & 5.7 & 6.5 & 1.2 & 0.5 & 0.05 & 2.8 & 9.30 & 81 \\
\hline $2 \mathrm{Ab3}$ & 6.3 & 28.8 & 14.7 & 22.4 & 1.6 & 0.2 & 0.07 & 8.8 & 10.02 & 92 \\
\hline $2 \mathrm{C3}$ & 1.3 & 13.6 & 8.2 & 12.3 & 1.6 & 0.1 & - & 4.9 & 9.63 & 54 \\
\hline \multicolumn{11}{|l|}{ Pedon 4, $3000 \mathrm{~m}$ asl } \\
\hline C1 & 0.4 & $\begin{array}{r}10.2 \\
2.7\end{array}$ & 1.5 & 2.3 & 1.0 & $\mathbf{0 . 5}$ & 0.00 & $\begin{array}{c}2.8 \\
<1\end{array}$ & $\begin{array}{r}10.39 \\
8.22\end{array}$ & $\begin{array}{l}61 \\
13\end{array}$ \\
\hline C2 & 0.2 & 1.7 & 1.0 & 1.5 & - & - & - & $<1$ & 7.93 & 8 \\
\hline C3 & 0.2 & 1.1 & 0.8 & 0.9 & - & - & - & $<\mathbf{1}$ & 7.82 & 7 \\
\hline 2Ab1 & 1.7 & 2.0 & 1.3 & 0.3 & - & - & 0.12 & $<1$ & 8.01 & 14 \\
\hline $2 \mathrm{Ab2}$ & 4.1 & 6.6 & 2.6 & 2.5 & 1.0 & 0.6 & 0.09 & 1.3 & 8.27 & 39 \\
\hline $\mathbf{2 A B b}$ & 2.8 & $\mathbf{7 . 3}$ & 2.5 & 4.5 & 1.8 & 0.4 & - & 1.8 & 8.07 & 38 \\
\hline $2 B w b$ & 2.0 & 8.1 & 2.7 & 6.1 & 2.3 & 0.2 & - & 2.7 & 8.04 & 28 \\
\hline $2 \mathrm{C} 4$ & 1.7 & 5.2 & 1.9 & 3.5 & 1.9 & 0.3 & - & 1.3 & 8.28 & 20 \\
\hline $2 \mathrm{C} 5$ & 0.4 & 2.6 & 1.4 & 2.2 & - & - & - & $<1$ & 8.08 & 6 \\
\hline \multicolumn{11}{|l|}{ Pedon 5, $2950 \mathrm{~m}$ asl } \\
\hline $\begin{array}{l}\text { A1 } \\
\mathbf{A}_{2}\end{array}$ & 3.0 & 7.2 & $\begin{array}{l}2.7 \\
3.1\end{array}$ & $\begin{array}{l}4.2 \\
3.8\end{array}$ & 1.6 & 0.4 & $\begin{array}{l}0.04 \\
0.05\end{array}$ & 1.6 & $\begin{array}{l}9.40 \\
9.72\end{array}$ & $\begin{array}{l}46 \\
46\end{array}$ \\
\hline A3 & $\begin{array}{l}3.4 \\
1.8\end{array}$ & 3.2 & $\begin{array}{l}3.1 \\
1.6\end{array}$ & $\begin{array}{l}3.0 \\
1.2\end{array}$ & $\begin{array}{l}1.5 \\
-\end{array}$ & $\begin{array}{c}0.3 \\
-\end{array}$ & $\begin{array}{l}0.09 \\
0.04\end{array}$ & $\begin{array}{l}1.9 \\
<1\end{array}$ & 8.42 & 20 \\
\hline A4 & 1.8 & 2.9 & 1.6 & 1.1 & - & - & 0.05 & $<\mathbf{1}$ & 8.55 & 18 \\
\hline AC1 & 0.8 & 1.9 & 1.2 & 1.1 & - & - & - & $<1$ & 7.86 & 11 \\
\hline AC2 & 0.4 & 1.3 & 0.9 & 0.9 & - & - & - & $<1$ & 7.81 & 6 \\
\hline C & 0.1 & 0.9 & 0.7 & 0.8 & - & - & - & $<\mathbf{1}$ & 7.70 & 3 \\
\hline \multicolumn{11}{|l|}{ Pedon $6,3060 \mathrm{~m}$ asl } \\
\hline $\mathbf{A}$ & 5.6 & 12.1 & 4.5 & 6.5 & 1.5 & 0.5 & 0.04 & 2.7 & 10.54 & 74 \\
\hline Bw & 1.9 & 6.0 & 3.2 & 4.1 & 1.3 & 0.3 & - & 1.9 & 9.87 & 37 \\
\hline BC & 0.7 & 3.1 & 2.0 & 2.4 & 1.3 & 0.2 & - & 1.2 & 9.24 & 15 \\
\hline C & 0.4 & 2.5 & 1.7 & 2.1 & 1.3 & 0.2 & - & 1.0 & 9.00 & 11 \\
\hline
\end{tabular}

Continued next page.

are particularly high in buried A horizons of high-elevation pedons (Table 3 ).

The molar ratios of $\mathrm{Al}_{\mathrm{p}} /$ organic $\mathrm{C}$ were calculated for recent and buried A horizons (Table 3 ). The $\mathrm{Al}_{\mathrm{p}} / \mathrm{C}$ ratios of recent $\mathrm{A}$ horizons are significantly higher in pedons above $2700 \mathrm{~m}$ asl than in pedons below $(\alpha=0.05)$, and buried $\mathrm{A}$ horizons generally have higher $\mathrm{Al}_{\mathrm{p}} / \mathrm{C}$ ratios than recent $\mathrm{A}$ horizons. The $2 \mathrm{Ab} 2$ and $3 \mathrm{Ab}$ horizons of Pedon 10 and the $3 \mathrm{Ab}$ horizon of Pedon 11 exhibit particularly high $\mathrm{Al}_{\mathrm{p}} / \mathrm{C}$ ratios $(0.16-0.19)$. Aluminum appears to be bound very strongly to organic functional groups in these horizons, exceeding the extraction power of ammonium oxalate-oxalic acid, which results in negative values of $\mathrm{Al}_{\mathrm{o}}-\mathrm{Al}_{\mathrm{p}}$ (Table 3 ).
The clay fractions of the studied soils exhibit a single IR absorption maximum around $1000 \mathrm{~cm}^{-1}$ (data not shown) and show no dehydroxylation between 300 and $400^{\circ} \mathrm{C}$ (Fig. 2), which rules out the presence of imogolite. Therefore, $\mathrm{Si}_{\mathrm{o}}$ is assumed to originate from allophane, although some of it may arise from incongruent dissolution of poorly ordered minerals, such as embryonic halloysite (Wada and Kakuto, 1985). In the studied soils, allophane contents increase with altitude and are highest in the three highest-elevation pedons (above $3200 \mathrm{~m}$ asl), where significant amounts are found in the entire profile. In pedons at intermediate elevations (3200$2700 \mathrm{~m}$ asl), allophane is found in smaller quantities and predominantly in the upper part of the profile, and 
Table 3. Continued.

\begin{tabular}{|c|c|c|c|c|c|c|c|c|c|c|}
\hline Horizon & $\mathbf{A l}_{\mathbf{p}} \dagger$ & $\mathbf{A l}_{0}+$ & $\mathbf{S i}_{0} \ddagger$ & $\mathbf{A} \mathbf{l}_{\mathbf{o}}-\mathbf{A} \mathbf{l}_{\mathrm{p}}$ & $\left(\mathbf{A} \mathbf{l}_{0}-\mathbf{A} \mathbf{l}_{\mathbf{p}}\right) / \mathbf{S i}_{0}$ & $\mathbf{A} \mathbf{l}_{\mathrm{p}} / \mathbf{A l}_{0}$ & $\mathbf{A} \mathbf{l}_{\mathrm{p}} / \mathbf{C}$ & Allophane & $\mathrm{pH}(\mathrm{NaF})$ & $\mathrm{PO}_{4}$ retention \\
\hline & & 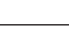 & $\mathbf{k g}^{-1}-$ & - & - & $\operatorname{lar}-$ & - & $\%$ & & $\%$ \\
\hline \multicolumn{11}{|l|}{ Pedon 7, $2900 \mathrm{~m}$ asl $§$} \\
\hline $\mathbf{A 2}$ & 2.4 & 9.1 & 4.9 & 6.7 & 1.4 & 0.3 & 0.05 & 2.9 & 10.23 & 48 \\
\hline $\mathbf{A 3}$ & 1.1 & 5.4 & 3.5 & 4.3 & 1.3 & 0.2 & 0.06 & 2.1 & 8.98 & 27 \\
\hline Bw & 0.5 & 3.7 & 2.8 & 3.2 & 1.2 & 0.1 & - & 1.4 & 8.54 & 14 \\
\hline C1 & 0.3 & 2.3 & 1.7 & 2.0 & - & - & - & $<1$ & 8.03 & 8 \\
\hline C2 & 0.2 & 1.4 & 0.9 & 1.2 & - & - & - & $<1$ & 7.90 & 4 \\
\hline $\mathbf{C 3}$ & 0.3 & 1.1 & 0.7 & 0.9 & - & - & - & $<1$ & 7.78 & 3 \\
\hline C4 & 0.2 & 1.0 & 0.8 & 0.8 & - & - & - & $<1$ & 7.71 & 1 \\
\hline \multicolumn{11}{|l|}{ Pedon $8,2740 \mathrm{~m}$ asl } \\
\hline $\begin{array}{l}\text { Ap } \\
\mathbf{A 1}\end{array}$ & $\begin{array}{l}1.7 \\
2.6\end{array}$ & $\begin{array}{l}4.2 \\
5.9\end{array}$ & $\begin{array}{l}1.7 \\
2.2\end{array}$ & $\begin{array}{l}2.5 \\
3.3\end{array}$ & $\begin{array}{l}1.6 \\
1.6\end{array}$ & $\begin{array}{l}0.4 \\
0.4\end{array}$ & $\begin{array}{l}0.03 \\
0.05\end{array}$ & $\begin{array}{l}1.0 \\
1.3\end{array}$ & $\begin{array}{l}9.16 \\
9.79\end{array}$ & $\begin{array}{l}26 \\
38\end{array}$ \\
\hline $\mathbf{A 2}$ & 2.0 & 6.5 & 3.3 & 4.5 & 1.4 & 0.3 & 0.05 & 2.0 & 9.54 & 36 \\
\hline Bw & 0.5 & 2.8 & 1.7 & 2.3 & 1.4 & 0.2 & - & 1.0 & 7.96 & 8 \\
\hline C & 0.1 & 1.2 & 1.1 & 1.1 & - & - & - & $<\mathbf{1}$ & 7.78 & $\mathbf{0}$ \\
\hline \multicolumn{11}{|l|}{ Pedon $9,2570 \mathrm{~m}$ asl } \\
\hline Ap & 0.2 & 1.6 & 0.9 & 1.4 & - & - & 0.01 & $<\mathbf{1}$ & 8.68 & 4 \\
\hline${ }_{\mathbf{B}}^{\mathbf{A}}$ & 0.2 & 1.5 & 0.8 & 1.3 & - & - & 0.02 & $<1$ & 8.54 & 4 \\
\hline Bw & 0.4 & 2.1 & 1.3 & 1.8 & - & - & - & $<\mathbf{1}$ & 9.04 & 9 \\
\hline $2 \mathbf{A b}$ & 0.4 & 2.3 & 1.4 & 1.9 & - & - & 0.04 & $<1$ & 8.82 & 7 \\
\hline $2 \mathrm{C} 1$ & 0.1 & 1.2 & 0.8 & 1.0 & - & - & - & $<\mathbf{1}$ & 8.09 & 1 \\
\hline $\mathbf{3 A B b}$ & 0.2 & 2.1 & 1.3 & 1.9 & - & - & - & $<\mathbf{1}$ & 8.86 & 6 \\
\hline $4 A b 1$ & 0.5 & 3.6 & 2.1 & 3.1 & 1.6 & 0.1 & 0.04 & 1.2 & 9.10 & 11 \\
\hline $4 \mathrm{Ab2} 2$ & 0.4 & 3.3 & 2.0 & 2.9 & 1.5 & 0.1 & 0.04 & 1.2 & 9.14 & 13 \\
\hline 4Bwb1 & 0.3 & 2.6 & 1.6 & 2.3 & - & - & - & $<1$ & 8.50 & 7 \\
\hline 4Bwb2 & 0.2 & 1.9 & 1.2 & 1.7 & - & - & - & $<1$ & 8.62 & 6 \\
\hline $4 B w b 3$ & 0.3 & 2.0 & 1.1 & 1.8 & - & - & - & $<\mathbf{1}$ & 8.81 & 4 \\
\hline $4 \mathrm{C} 2$ & 0.2 & 1.3 & 0.9 & 1.1 & - & - & - & $<\mathbf{1}$ & 8.51 & 3 \\
\hline \multicolumn{11}{|l|}{ Pedon $10,2560 \mathrm{~m}$ asl } \\
\hline A & 0.2 & 1.9 & 1.0 & 1.7 & - & - & 0.03 & $<\mathbf{1}$ & 8.17 & 7 \\
\hline C1 & 0.0 & 0.8 & 0.6 & 0.8 & - & - & - & $<1$ & 7.86 & 3 \\
\hline C2 & 0.0 & 1.3 & 1.1 & 1.3 & - & - & - & $<\mathbf{1}$ & 7.86 & 1 \\
\hline C3 & 0.1 & 0.9 & 0.8 & 0.9 & - & - & - & $<\mathbf{1}$ & 7.83 & 1 \\
\hline C4 & 0.1 & 0.8 & 0.6 & 0.8 & - & - & - & $<\mathbf{1}$ & 7.89 & 3 \\
\hline C5 & 0.1 & 1.0 & 0.8 & 0.9 & - & - & - & $<\mathbf{1}$ & 8.05 & 1 \\
\hline C6 & 0.2 & 1.2 & 0.9 & 1.0 & - & - & - & $<\mathbf{1}$ & 8.01 & 3 \\
\hline C7 & 0.1 & 0.9 & 0.8 & 0.8 & - & - & - & $<1$ & 7.90 & 0 \\
\hline C8 & 0.2 & 1.0 & 0.7 & 0.9 & - & - & - & $<1$ & 8.09 & 3 \\
\hline C9 & 0.1 & 0.9 & 0.7 & 0.8 & - & - & - & $<1$ & 7.80 & 1 \\
\hline C10 & 0.1 & 0.7 & 0.7 & 0.6 & - & - & - & $<1$ & 7.79 & 1 \\
\hline C11 & 0.1 & 0.9 & 1.0 & 0.8 & - & - & - & $<\mathbf{1}$ & 7.85 & 0 \\
\hline C12 & 0.1 & 1.0 & 1.0 & 1.0 & - & - & - & $<1$ & 7.78 & 0 \\
\hline $2 \mathrm{Ab} 1$ & 0.3 & 1.0 & 0.7 & 0.7 & - & - & 0.02 & $<\mathbf{1}$ & 7.89 & 3 \\
\hline $2 \mathrm{Ab2}$ & 2.0 & 1.8 & 1.1 & -0.2 & - & - & 0.16 & $<1$ & 8.21 & 11 \\
\hline $2 \mathrm{C13}$ & 0.3 & 2.2 & 1.5 & 1.9 & - & - & - & $<\mathbf{1}$ & 8.18 & 6 \\
\hline 3Ab & 6.7 & 2.3 & 1.1 & -4.4 & - & - & 0.17 & $<1$ & 8.34 & 15 \\
\hline \multicolumn{11}{|l|}{ Pedon $11,2630 \mathrm{~m}$ asl } \\
\hline $\mathbf{A}$ & 0.3 & 1.5 & 0.9 & 1.2 & - & - & 0.02 & $<\mathbf{1}$ & 8.49 & 6 \\
\hline C1 & 0.2 & 1.6 & 1.0 & 1.4 & - & - & - & $<\mathbf{1}$ & 8.51 & 0 \\
\hline C2 & 0.2 & 0.9 & 0.7 & 0.7 & - & - & - & $<\mathbf{1}$ & 8.04 & 1 \\
\hline $2 \mathbf{A b}$ & 1.1 & 1.6 & 1.0 & 0.4 & - & - & 0.10 & $<1$ & 8.22 & 7 \\
\hline $2 \mathrm{C3}$ & 0.9 & 1.7 & 1.0 & 0.8 & - & - & - & $<1$ & 8.20 & 7 \\
\hline $\mathbf{3 A b}$ & 6.4 & 2.5 & 1.1 & -4.0 & - & - & 0.19 & $<\mathbf{1}$ & 8.50 & 17 \\
\hline \multicolumn{11}{|l|}{ Pedon $12,2410 \mathrm{~m}$ asl } \\
\hline $\mathbf{A 2}$ & 0.1 & 1.0 & $\begin{array}{l}0.0 \\
0.9\end{array}$ & 1.2 & - & - & $\begin{array}{l}0.01 \\
0.01\end{array}$ & $\begin{array}{l}<\mathbf{1} \\
<\mathbf{1}\end{array}$ & $\begin{array}{l}7.91 \\
7.89\end{array}$ & $\begin{array}{l}6 \\
6\end{array}$ \\
\hline $\mathbf{A C}$ & 0.1 & 1.1 & 0.7 & 1.0 & - & - & - & $<\mathbf{1}$ & 8.00 & 4 \\
\hline C1 & 0.0 & 0.9 & 0.7 & 0.8 & - & - & - & $<1$ & 8.00 & 3 \\
\hline C2 & 0.1 & 1.0 & 0.7 & 0.9 & - & - & - & $<\mathbf{1}$ & 7.97 & 4 \\
\hline $\mathbf{C 3}$ & 0.0 & 0.7 & 0.5 & 0.7 & - & - & - & $<1$ & 8.13 & 4 \\
\hline 2Ab1 & 0.1 & 1.4 & 0.8 & 1.3 & - & - & 0.01 & $<\mathbf{1}$ & 8.40 & 6 \\
\hline $2 \mathrm{Ab2}$ & 0.1 & 1.2 & 0.7 & 1.1 & - & - & 0.01 & $<1$ & 8.49 & 6 \\
\hline $2 \mathrm{C} 4$ & 0.1 & 1.2 & 0.8 & 1.1 & - & - & - & $<\mathbf{1}$ & 8.54 & 7 \\
\hline
\end{tabular}

$\dagger$ p, pyrophosphate-extractable.

$\leftarrow$ o, acid oxalate-extractable.

$\S$ asl, above sea level.

in low-elevation pedons (below $2700 \mathrm{~m}$ asl), allophane contents are generally below $1 \%$ (Table 3 ).

The molar ratios of $\mathrm{Al}_{\mathrm{p}} / \mathrm{Al}_{\mathrm{o}}$, calculated for soil horizons with $\geq 1 \%$ allophane (Table 3 ), reveal that in recent A horizons of high-elevation pedons, the majority of active aluminol groups pertains to Al-humus complexes $\left(\mathrm{Al}_{\mathrm{p}} / \mathrm{Al}_{\mathrm{o}}>0.5\right)$, whereas in subsurface horizons including buried A horizons, the majority of active $\mathrm{Al}-\mathrm{OH}$ groups appears to be associated with allophane $\left(\mathrm{Al}_{\mathrm{p}} / \mathrm{Al}_{\mathrm{o}}<0.5\right)$.

\section{Crystalline Colloidal Constituents}

Pedons 2, 3, and 4, representing a toposequence on the slopes of Cerro Huanansí (Table 1, Fig. 1), were 


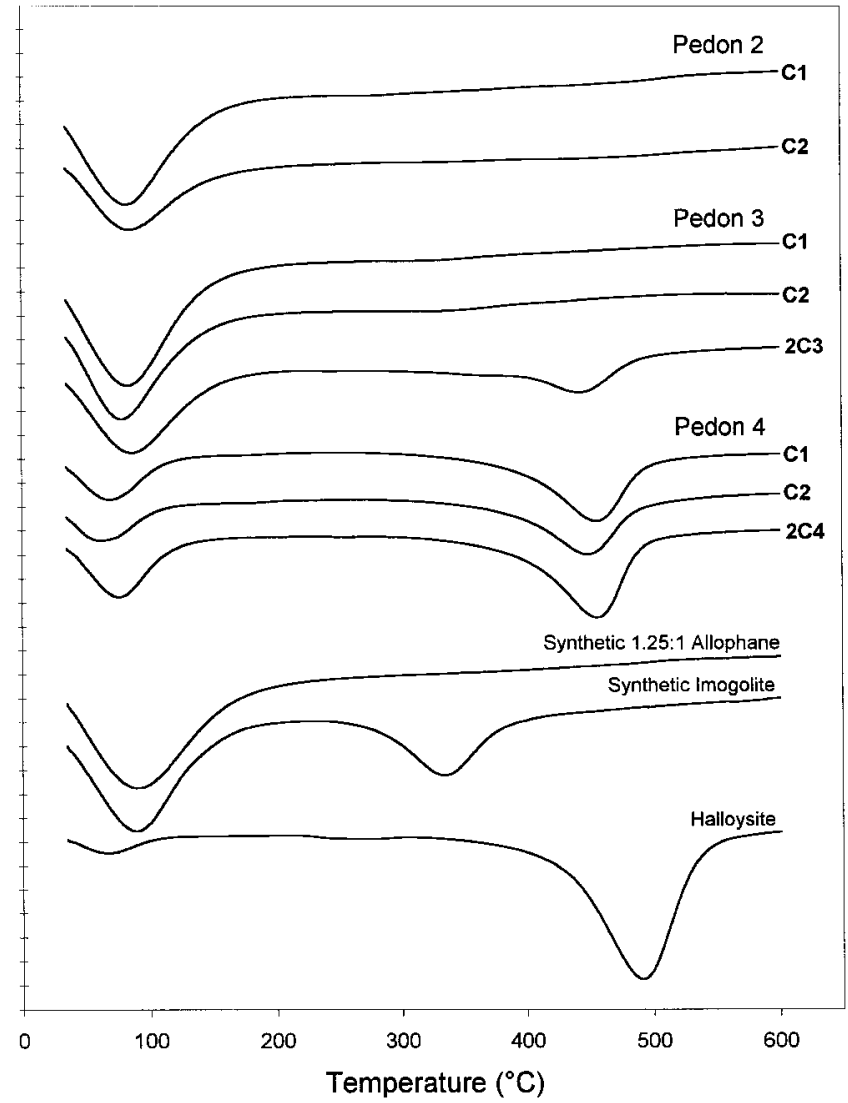

Fig. 2. Differential scanning calorimetry graphs of selected soil horizons and pure materials; one increment on the $\boldsymbol{y}$-axis corresponds to a heat flow of $0.2 \mathrm{~W} \mathrm{~g}^{-1}$.

selected to further study the mineralogy of the soil colloidal fraction at elevations ranging from 3000 to $3900 \mathrm{~m}$ asl. The clay fractions of $\mathrm{C}$ horizons from recent soils and paleosols were analyzed with DSC (Fig. 2) and XRD (Fig. 3 and 4).

In recent $\mathrm{C}$ horizons of Pedons 2 and 3 (3900 and $3400 \mathrm{~m}$ asl, respectively), a large endothermic peak between 35 and $150^{\circ} \mathrm{C}$ was found, which is attributed to the removal of large amounts of adsorbed water, presumably associated with allophane, but no further DSC peaks were detected above $150^{\circ} \mathrm{C}$ (Fig. 2). On the other hand, recent $\mathrm{C}$ horizons of Pedon 4 (3000 m asl) show a rather small water peak between 35 and $100^{\circ} \mathrm{C}$, but exhibit a distinct endothermic peak between 400 and $500^{\circ} \mathrm{C}$, which is larger in the $\mathrm{C} 1$ than in the $\mathrm{C} 2$ horizon. In the paleosol, Pedon 3 shows a small and Pedon 4 a large endothermic peak between 400 and $500^{\circ} \mathrm{C}$ (Fig. 2). This peak is considered to arise from the dehydroxylation of kaolin minerals, such as halloysite. It occurs at lower temperatures in the studied clay samples than in the reference pure halloysite sample (Fig. 2), which may be due to smaller crystal sizes in the studied clays.

The XRD patterns of Pedons 2 and 3 reveal that the $\mathrm{C} 1$ horizons of both pedons are devoid of clay minerals (Fig. 3). However, in the $\mathrm{C} 2$ horizons, a prominent reflection at $1.4 \mathrm{~nm}$ was found with $\mathrm{Mg}$ saturation, part of which expanded on ethylene glycol (EG) solvation. Saturation with K resulted in partial collapse of this reflection, which gradually shifted toward $1 \mathrm{~nm}$ on heating to 110,300 , and $550^{\circ} \mathrm{C}$ (Fig. 3). This indicates the presence of smectitic (and possibly vermiculitic) layers with partial hydroxy-Al interlayering. The peak at $0.7 \mathrm{~nm}$ is believed to correspond with the 002 reflections from the 1.4-nm phases, since the DSC data of these horizons did not indicate the presence of kaolin minerals (Fig. 2).

The $\mathrm{C} 1$ horizon of Pedon 4 exhibits a 1.4-nm reflection with $\mathrm{Mg}$ saturation that expands on EG solvation and collapses to $1 \mathrm{~nm}$ with $\mathrm{K}$ saturation, and is therefore considered to pertain to smectite (Fig. 3). The same horizon further shows a broad reflection between 0.72 and $1 \mathrm{~nm}$, part of which expands to near $1.1 \mathrm{~nm}$ when solvated with EG. The reflection sharpens and collapses to near $0.72 \mathrm{~nm}$ when heated to 110 and $300^{\circ} \mathrm{C}$ and disappears when further heated to $550^{\circ} \mathrm{C}$ (Fig. 3). This is attributed to the presence of two populations of halloysite, that is, the $7 \AA$ and the hydrated $10 \AA$ forms, respectively. The $\mathrm{C} 2$ horizon of Pedon 4 shows a similar XRD pattern, indicating the presence of smectite and halloysite. However, the reflections are weaker and the collapsed halloysite peak (after heating to 110 and $300^{\circ} \mathrm{C}$ ) is broader than in the horizon above (Fig. 3). This suggests that the crystallinity of halloysite decreases from the $\mathrm{C} 1$ to the underlying $\mathrm{C} 2$ horizon of Pedon 4.

In the paleosol, smectite was not detected; however, both 7 and $10 \AA$ halloysite was found in Pedon 3 (Fig. 4), and only hydrated $(10 \AA)$ halloysite was found in Pedon 4 (Fig. 4). The collapsed halloysite peaks are sharper in the paleosols than in the recent soils (Fig. 3 and 4), indicating higher crystallinity in the former.

The presence of halloysite in recent soils and paleosols of pedons below $3000 \mathrm{~m}$ asl was further confirmed by XRD analysis (data not shown). A diffuse, broad hump above $20^{\circ} 2 \theta$ observed in the XRD patterns of the studied clay samples is ascribed to the presence of pyroclastic glass.

\section{DISCUSSION}

\section{Genesis and Classification of Recent Soils}

With an age of approximately $3000 \mathrm{yr}$, the soils formed on the recent volcanic deposits of the study area are considered to be in their early stages of development. This is also evident from their generally low clay contents and the abundance of primary minerals and pyroclastic glass. However, there appear to be dramatic altitudinal differences as to the path and rate at which pedogenesis proceeds in the area under study. Key soil properties of recent A horizons, such as organic $\mathrm{C}$ content, $\mathrm{Al}_{0}$, and $\mathrm{PO}_{4}$ retention, are significantly correlated with elevation $\left(r=0.93^{* * *}, 0.83^{* * *}\right.$, and $0.90^{* * *}$, respectively) and suggest grouping of the studied pedons into three elevation zones, that is, a high zone above $3200 \mathrm{~m}$ asl, an intermediate zone between 3200 and $2700 \mathrm{~m}$ asl, and a low zone below $2700 \mathrm{~m}$ asl. The abovementioned soil properties are significantly different $(\alpha=0.05)$ between these three zones. The observed 

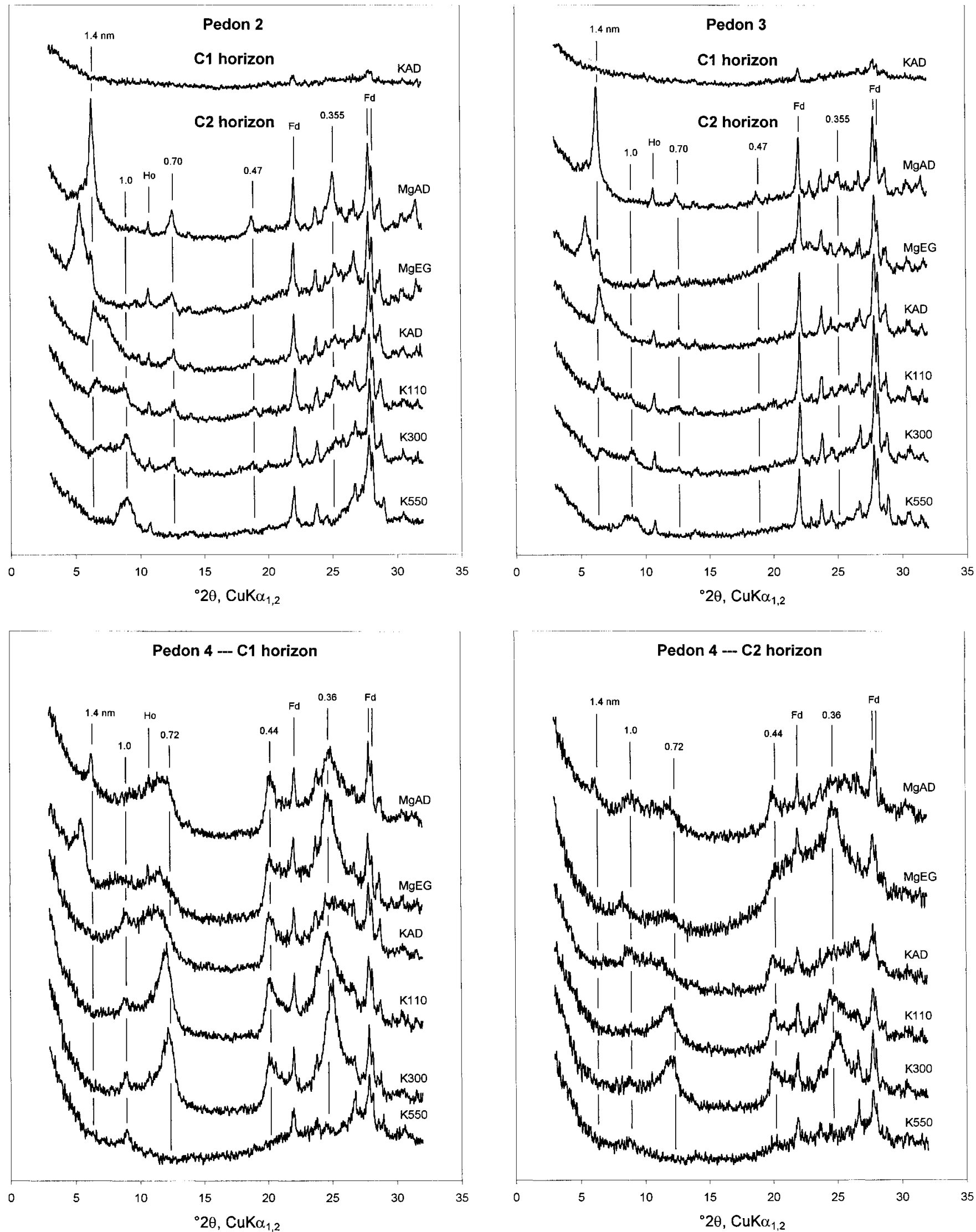

Fig. 3. X-ray diffraction patterns of selected recent soils; MgAD and MgEG: Mg saturation, air-dried, and ethylene glycol solvated, respectively; $\mathrm{KAD}$, K110, K300, and K550: K saturation, air-dried, and heated to 110,300 , and $550^{\circ} \mathrm{C}$, respectively; Ho: hornblende; Fd: feldspar. 

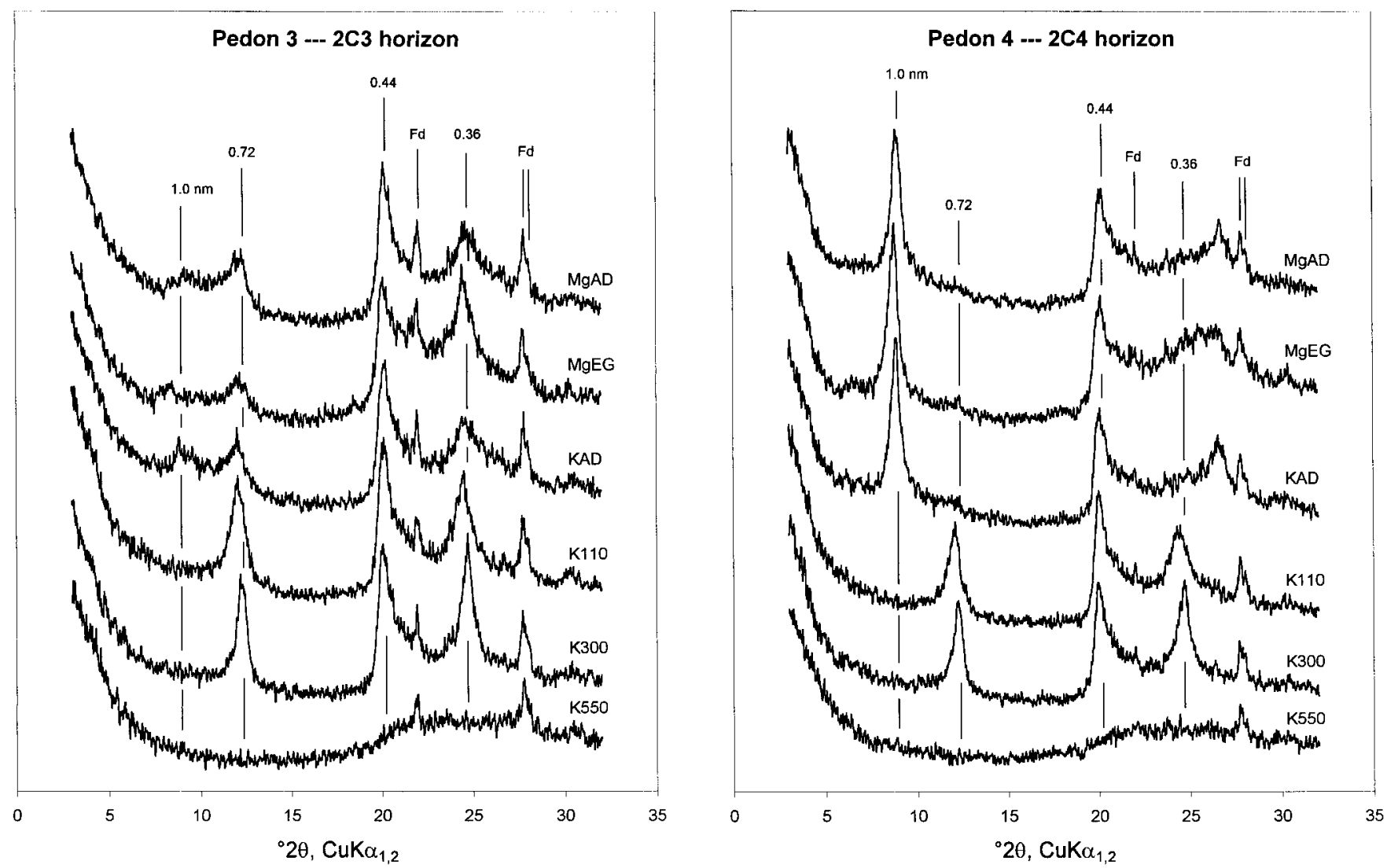

Fig. 4. X-ray diffraction patterns of selected paleosols; MgAD and MgEG: Mg saturation, air-dried, and ethylene glycol solvated, respectively; KAD, K110, K300, and K550: K saturation, air-dried, and heated to 110,300 , and $550^{\circ} \mathrm{C}$, respectively; Fd: feldspar.

altitudinal trends correspond with climatic differences as determined by elevation on the volcano; however, these differences are interrelated with differences in land use and vegetation. At higher elevations, higher rainfall and lower evapotranspiration (ET) caused by lower temperatures and higher humidity, result in greater leaching and less pronounced seasonal drying. As precipitation decreases and ET increases with decreasing elevation, the soils experience less leaching and a pronounced period of desiccation during the dry season.

The high-leaching environment at elevations above $3200 \mathrm{~m}$ asl has resulted in the formation of andic soil properties, as defined by Soil Survey Staff (1998) and manifested by the dominant presence of active aluminol groups associated with allophane and Al-humus complexes as well as by high $\mathrm{PO}_{4}$ retention. High additions of organic matter from the páramo vegetation combined with slowed decomposition due to low temperatures, low $\mathrm{pH}$ values, and the presence of stabilizing Al-humus complexes may have caused the increased accumulation of organic $\mathrm{C}$ and the formation of melanic epipedons at these elevations. The soils are classified as Melanocryands and Melanudands, which due to their relatively weak development and comparatively low contents of weathering products belong to the Vitric and Pachic Vitric subgroups, respectively (Table 1). The colloidal fraction of these soils is dominated by amorphous constituents and characterized by the absence of halloysite. The predominance of Al-humus complexes over allo- phane in the uppermost horizons of the highest-elevation pedons is believed to be mainly a result of $\mathrm{pH}$ $\left(\mathrm{H}_{2} \mathrm{O}\right)$ values $<5$ in these horizons, which have been shown to inhibit the formation of allophane and favor that of Al-humus complexes (Shoji et al., 1982; Shoji and Fujiwara, 1984). The increased presence of organic ligands in these horizons may have further impeded the precipitation of allophane by competition with orthosilicic acid for soluble Al.

Evidence of 2:1-type phyllosilicates with partial hydroxy- $\mathrm{Al}$ interlayering was found in the $\mathrm{C} 2$ horizons of Pedons 2 and 3 , whereas the overlying $\mathrm{C} 1$ horizons as well as the underlying $\mathrm{C} 3$ horizon (Pedon 2) did not contain clay minerals (Fig. 3). Since no A horizons were found in between the three tephra layers, they are considered to arise from consecutive eruptions of more or less the same age. Because of the isolated occurrence of 2:1-type phyllosilicates in only the $\mathrm{C} 2$ horizons, it is believed that incorporation during eruption, as reported by Dudas and Harward (1975), Pevear et al. (1982), and Ping et al. (1988), is the likely origin of the observed clay mineral association. The presence of expandable silicate layers may have counteracted the formation of allophane by competition with orthosilicic acid for hydroxy-Al polymers, thus resulting in lower allophane contents in the $\mathrm{C} 2$ horizons than in the $\mathrm{C} 1$ horizons above (Table 3 ).

As leaching decreases with decreasing elevation, andic soil properties become less strongly expressed and 
are confined to the upper horizons in pedons between 3200 and $2700 \mathrm{~m}$ asl, disappearing completely in pedons below $2700 \mathrm{~m}$ asl. Organic matter contents decrease with decreasing elevation, presumably because of less additions in cultivated land and more rapid decomposition due to higher temperatures, higher $\mathrm{pH}$ values, and lower amounts of stabilizing Al-humus complexes. This has resulted in the formation of umbric epipedons at intermediate elevations (3200-2700 m asl) and ochric epipedons containing $<1 \%$ organic $\mathrm{C}$ at elevations below $2700 \mathrm{~m}$ asl. At intermediate elevations, the soils are classified as Humic Udivitrands with the exception of Pedon 4, which has andic soil properties in the A horizon, but does not meet the depth requirement for Andisols (Soil Survey Staff, 1998) and is therefore classified in the Andic subgroup of Inceptisols (Table 1). At elevations below $2700 \mathrm{~m}$ asl, the soils are classified as Dystrudepts in the presence, and Udorthents in the absence of a Bw horizon, all belonging to the Vitrandic subgroup due to high contents of pumice fragments and pyroclastic glass (Table 1 ).

Pedon 4 is located on the lower backslope of Cerro Huanansí, with Pedons 2 and 3 located close to the summit and on the middle part of the backslope, respectively, and Pedon 5 just $50 \mathrm{~m}$ below Pedon 4, on the upper part of a depositional fan with considerably lower slope gradient (Fig. 1). The influence of topography on pedogenesis has been shown by Chen et al. (1999), who studied a toposequence of volcanic ash soils in subtropical Taiwan and attributed thinner sola and lower topsoil organic matter contents in backslope positions to increased lateral water flow and surface runoff. The toposequence of Cerro Huanansí demonstrates the impact of topography on soil development in the area under study. While the summit pedon has two A horizons reaching to a depth of $100 \mathrm{~cm}$, the topsoils are considerably shallower (40 and $30 \mathrm{~cm}$ ) in the middle and lower backslope positions, respectively (Table 2 ). This is likely the result of translocation of topsoil material downslope, which has been deposited where the gradient shows a sharp decline, as evident from the presence of four A horizons reaching to a depth of $150 \mathrm{~cm}$ in Pedon 5 (Table 2). Thus, whereas Pedons 2, 3, and 5 are classified as Andisols, the removal of andic soil properties along with the erosion of topsoil material results in the classification of Pedon 4 as an Inceptisol.

As a result of drier conditions, halloysite becomes increasingly dominant in the colloidal fraction of pedons below $3200 \mathrm{~m}$ asl, whereas allophane and Al-humus complexes only occur in the upper profile of pedons between 3200 and $2700 \mathrm{~m}$ asl and are absent below $2700 \mathrm{~m}$ asl. Larger amounts and higher crystallinity of halloysite in the $\mathrm{C} 1$ than in the underlying $\mathrm{C} 2$ horizon of Pedon 4 (Fig. 2 and 3) suggest pedogenic origin with enhanced weathering closer to the soil surface. The coexistence of allophane and halloysite in recent soils at intermediate elevation marks a transition from the allophane-dominated soils at high elevation to the halloysite-dominated soils at low elevation. Leaching during the rainy season appears to lower soil solution Si activity sufficiently in the upper profile and thus trigger the formation of allo- phane in the upper soil horizons of this transition zone, whereas desiccation during the dry season may promote the formation of halloysite. Similar differential formation of amorphous and crystalline weathering products with fluctuating leaching and desiccation periods has been proposed by Takahashi et al. (1993) for volcanic ash soils in the xeric moisture regime of northern California.

Elevated topsoil clay contents in pedons above $2700 \mathrm{~m}$ asl may be the result of a more aggressive weathering environment involving greater leaching and diurnal freeze-thaw cycles; however, part of the clay fraction may arise from minor additions of fine-textured tephra from surrounding volcanoes, which may have been deposited in the higher-lying western part of the study area. The lower soil $\mathrm{pH}\left(\mathrm{H}_{2} \mathrm{O}\right)$ values at higher elevations are believed to arise from greater proton inputs with increased precipitation, greater leaching of basic cations, and greater activity of organic acids as a result of slowed organic matter decomposition.

\section{Pedogenesis of Paleosols}

Paleosols buried by the recent volcanic deposits are frequently encountered in the study area. Some mixing of new deposits with the old surface may have occurred upon deposition of recent tephra. This seems to be noticeable in Pedons 1 through 4, where the upper $\mathrm{Ab}$ horizons have significantly lower organic $\mathrm{C}$ contents than the Ab horizons underneath (Table 2). The pedogenesis of paleosols has been taking place over a longer time and under climatic conditions that may have deviated from those of the present day.

It has been shown for volcanic ash soils that humus evolves over time from forms with low complexing ability to forms with high complexing ability for $\mathrm{Al}$ (Wada, 1980). This appears to be the case in the paleosols of Pedons 10 and 11, which are highly indurated and presumably represent the oldest soils encountered in the study area. Higher clay contents and smaller amounts of primary minerals as compared with the recent soils reflect the prolonged exposure of paleosols to the processes of weathering. Nevertheless, the composition of their colloidal fraction shows virtually the same altitudinal sequence observed in the recent soils, with allophane and Al-humus complexes dominating at higher elevations and halloysite dominating at lower elevations. However, the contents of these constituents are higher in paleosols than in recent soils (Table 3, Fig. 2).

The highest allophane contents among the studied soils were found in paleosols above $3200 \mathrm{~m}$ asl (Table 3). This may reflect longer soil development as well as differences in parent material compared with the overlying recent soils. The paleosols of Pedons 1 and 2 are believed to have formed after the last glaciation, which reached as low as 3400 to $3600 \mathrm{~m}$ asl (Schubert and Clapperton, 1990), approximately 10000 to $12000 \mathrm{yr}$ ago. The paleosols of Pedons 3 and 4 have formed on tephra deposits that are believed to be associated with earlier eruptions from the volcanic complex of Cotacachi with an age of more than $40000 \mathrm{yr}$ (Hall and Mothes, 1994). In a road cut near Pedon 1, the buried 
A horizons were found to directly overlie what appear to be colluvial deposits of unweathered volcanic rocks. The abrupt boundary between this colluvium and the deepest $\mathrm{Ab}$ horizon and the absence of weathered rock fragments in the $\mathrm{Ab}$ horizon point to a paleosol parent material different from the colluvium, possibly glacial outwash deposited on top of the colluvium. In this case, the parent material could have been preweathered and already contained allophane at the beginning of postglacial pedogenesis in the early Holocene, which would explain the relatively high allophane contents in the 2Ab3 horizon of Pedon 1 (Table 3).

Wada (1980) reported interaction of soil organic matter with allophane in buried A horizons to result in the formation of stable silt- and sand-sized aggregates that are resistant to dispersion. The presence of such aggregates in the $\mathrm{Ab}$ horizons of high-elevation pedons is suggested by pronounced differences between measured and estimated clay contents (Table 2). In these horizons, complexation with humic substances may have stabilized and protected allophane against dissolution and thus fostered its accumulation over time. A significant fraction of the humus complexing sites may be associated with $\mathrm{Al}$ from allophanic structures, possibly through ligand exchange of carboxyl groups to aluminol surface groups (Harsh, 2000).

Small amounts of fairly crystalline halloysite were found in the 2C3 horizon of Pedon 3 (Fig. 2 and 4), which also contains significant amounts of allophane (Table 3 ). The presence of halloysite in the paleosol of this pedon may be explained by increased $\mathrm{Si}$ inputs due to leaching from the recent depositional overburden. In that case, the conditions favoring halloysite formation would have been established with the deposition of recent tephra about $3000 \mathrm{yr}$ BP. However, the halloysite found in the paleosol of Pedon 3 is clearly more crystalline than that found in the recent tephra of Pedon 4 (Fig. 3 and 4). This suggests that the former has formed over a longer time, possibly under different climatic conditions. In fact, pollen analyses in lacustrine sediments near the study area reveal evidence for a drier climate in the late-glacial period (Colinvaux et al., 1988), which could have resulted in the preferential formation of halloysite in Pedon 3. As the climate became moister at the beginning of the Holocene (Colinvaux et al., 1988), increased leaching may have lowered soil solution Si activities thus favoring the dissolution of halloysite and the precipitation of allophane.

\section{SUMMARY AND CONCLUSIONS}

In the area under study, three altitudinal zones can be distinguished with respect to soil formation; that is, a high zone above $3200 \mathrm{~m}$ asl, an intermediate zone between 3200 and $2700 \mathrm{~m}$ asl, and a low zone below $2700 \mathrm{~m}$ asl. In the high zone, the soil colloidal fraction is dominated by amorphous material and the soils are Andisols with melanic epipedons. In the low zone, halloysite is the predominant colloidal constituent and the soils are Inceptisols and Entisols. The intermediate zone is a transition zone in which allophane and halloysite coexist.
The soils of this zone are generally Andisols with umbric epipedons.

This altitudinal weathering sequence was observed in the 3000-yr-old recent soils and in paleosols considered older than $40000 \mathrm{yr}$, which shows that different time of soil development has not caused marked differences in the formation and transformation of colloidal weathering products.

Climate is considered the overriding factor responsible for the observed altitudinal differences in soil development. Differences in rainfall and evapotranspiration resulting in different leaching regimes are believed to have caused the differential formation of allophane or halloysite and thus the differential development of andic soil properties. Temperature has further affected organic matter decomposition causing increased accumulation with elevation and thus resulting in the altitudedependent formation of different epipedons.

There is some evidence that halloysite formed at higher elevations under the drier climatic conditions of the late-glacial. Thus, while the observed trends suggest altitudinal zonation with respect to soil formation, the boundaries between the zones may shift according to climatic changes over time. Moreover, the effects of topography on soil development may be superimposed on the general altitudinal trends, as observed on the steep-sloping parts of the study area, where andic soil properties have been removed from backslope positions along with the erosion of topsoil material.

\section{ACKNOWLEDGMENTS}

This study was made possible through support provided by the Office of Agriculture, Bureau for Economic Growth, Agriculture and Trade, U.S. Agency for International Development through the Sustainable Agriculture and Natural Resources Management Collaborative Research Support Program (SANREM CRSP) under the terms of Cooperative Agreement Number PCE-A-00-98-00019-00. The opinions expressed herein are those of the authors and do not necessarily reflect the views of the U.S. Agency for International Development. We thank Dr. Robert Rhoades, the project manager of SANREM-Andes, for his continuous support of our undertakings. We express our gratitude to Drs. Randy Dahlgren and James Harsh for kindly providing pure allophane and imogolite samples, and to Dr. Paul Schroeder for assistance with infrared spectroscopy and X-ray diffraction. We are particularly grateful to UNORCAC, the Unión de Organizaciones Campesinas e Indígenas de Cotacachi, and to the many local people who helped and supported us in Ecuador, and for the privilege of working in that unique land.

\section{REFERENCES}

Athens, J.S. 1999. Volcanism and archaeology in the northern highlands of Ecuador. p. 157-189. In P. Mothes (ed.) Actividad volcánica y pueblos precolombinos en el Ecuador. (In English.) Ediciones Abya-Yala, Quito, Ecuador.

Bakker, L., D.J. Lowe, and A.G. Jongmans. 1996. A micromorphological study of pedogenic processes in an evolutionary soil sequence formed on Late Quaternary rhyolitic tephra deposits, North Island, New Zealand. Quat. Int. 34-36:249-261.

Chartres, C.J., and C.F. Pain. 1984. A climosequence of soils on Late Quaternary volcanic ash in highland Papua New Guinea. Geoderma 32:131-155.

Chen, Z.-S., V.B. Asio, and D.-F. Yi. 1999. Characteristics and genesis 
of volcanic soils along a toposequence under a subtropical climate in Taiwan. Soil Sci. 164:510-525.

Colinvaux, P.A., K. Olson, and K.-B. Liu. 1988. Late-glacial and Holocene pollen diagrams from two endorheic lakes of the inter-Andean plateau of Ecuador. Rev. Palaeobot. Palynol. 55:83-99.

Drever, J.E. 1973. The preparation of oriented clay mineral specimens for x-ray diffraction analysis by a filter-membrane technique. Am. Mineral. 58:553-554.

Dudas, M.J., and M.E. Harward. 1975. Inherited and detrital 2:1 type phyllosilicates in soils developed from Mazama ash. Soil Sci. Soc. Am. Proc. 39:571-577.

Hall, M.L., and P.A. Mothes. 1994. Tefroestratigrafía holocénica de los volcanes principales del valle interandino, Ecuador. p. 47-67. In R. Marocco (ed.) El contexto geológico del espacio físico ecuatoriano: Neotectónica, geodinámica, volcanismo, cuencas sedimentarias, riesgo sísmico. Estudios de Geografía, vol. 6. (In Spanish.) Colegio de Geógrafos del Ecuador, Corporación Editora Nacional, Quito, Ecuador

Harsh, J. 2000. Poorly crystalline aluminosilicate clays. p. F169-F182. In M.E. Sumner (ed.) Handbook of soil science. CRC Press, Boca Raton, FL.

Hunter, C.R., and A.J. Busacca. 1989. Dispersion of three andic soils by ultrasonic vibration. Soil Sci. Soc. Am. J. 53:1299-1302.

Kimble, J.M., C.L. Ping, M.E. Sumner, and L.P. Wilding. 2000. Andisols. p. E209-E224. In M.E. Sumner (ed.) Handbook of soil science. CRC Press, Boca Raton, FL.

Mizota, C. 1983. Eolian origin of the micaceous minerals in an Ando soil from Kitakami, Japan. Soil Sci. Plant Nutr. 29:379-382.

Mizota, C., and Y. Takahashi. 1982. Eolian origin of quartz and mica in soils developed on basalts in northwestern Kyushu and San-in, Japan. Soil Sci. Plant Nutr. 28:369-378.

Mothes, P., and M.L. Hall. 1991. El paisaje interandino y su formación por eventos volcánicos de gran magnitud. p. 19-38. In P. Mothes (ed.) El paisaje volcánico de la sierra ecuatoriana: Geomorfología, fenómenos volcánicos y recursos asociados. Estudios de Geografía, vol. 4. (In Spanish.) Colegio de Geógrafos del Ecuador, Corporación Editora Nacional, Quito, Ecuador.

Mothes, P.A., and M.L. Hall. 1999. Quilotoa's 800 y BP ash: A valuable stratigraphic marker unit for the integration period. p. 111-138. In P. Mothes (ed.) Actividad volcánica y pueblos precolombinos en el Ecuador. (In English.) Ediciones Abya-Yala, Quito, Ecuador.

Nieuwenhuyse, A., P.S.J. Verburg, and A.G. Jongmans. 2000. Mineralogy of a soil chronosequence on andesitic lava in humid tropical Costa Rica. Geoderma 98:61-82.

Nizeyimana, E., T.J. Bicki, and P.A. Agbu. 1997. An assessment of colloidal constituents and clay mineralogy of soils derived from volcanic materials along a toposequence in Rwanda. Soil Sci. 162: 361-371.

Nouvelot, J.-F., P. Le Goulven, M. Alemán, and P. Pourrut. 1995. Análisis estadístico y regionalización de las precipitaciones en el Ecuador. p. 27-66. In P. Pourrut (ed.) El agua en el Ecuador: Clima, precipitaciones, escorrentía. Estudios de Geografía, vol. 7. (In Spanish.) ORSTOM, Colegio de Geógrafos del Ecuador, Corporación Editora Nacional, Quito, Ecuador.

Parfitt, R.L. 1990. Allophane in New Zealand-A review. Aust. J. Soil Res. 28:343-360

Parfitt, R.L., M. Russell, and G.E. Orbell. 1983. Weathering sequence of soils from volcanic ash involving allophane and halloysite, New Zealand. Geoderma 29:41-57.

Parfitt, R.L., M. Saigusa, and J.D. Cowie. 1984. Allophane and halloysite formation in a volcanic ash bed under different moisture conditions. Soil Sci. 138:360-364.

Parfitt, R.L., and A.D. Wilson. 1985. Estimation of allophane and halloysite in three sequences of volcanic soils, New Zealand. p. 1-8. In E. Fernandez Caldas and D.H. Yaalon (ed.) Volcanic soils: Weathering and landscape relationships of soils on tephra and basalt. Catena Suppl. 7. Catena Verlag, Cremlingen, Germany.

Pevear, D.R., D.P. Dethier, and D. Frank. 1982. Clay minerals in the 1980 deposits from Mount St. Helens. Clays Clay Miner. 30:241-252.

Ping, C.L., S. Shoji, and T. Ito. 1988. Properties and classification of three volcanic ash-derived pedons from Aleutian Islands and Alaska peninsula, Alaska. Soil Sci. Soc. Am. J. 52:455-462.

Schubert, C., and C.M. Clapperton. 1990. Quaternary glaciations in the northern Andes (Venezuela, Colombia and Ecuador). Quat. Sci. Rev. 9:123-135.

Shoji, S., and Y. Fujiwara. 1984. Active $\mathrm{Al}$ and $\mathrm{Fe}$ in the humus horizons of Andosols from northeastern Japan: Their forms, properties, and significance in clay weathering. Soil Sci. 137:216-226.

Shoji, S., Y. Fujiwara, I. Yamada, and M. Saigusa. 1982. Chemistry and clay mineralogy of Ando soils, Brown Forest soils, and Podzolic soils formed from recent Towada ashes, northeastern Japan. Soil Sci. 133:69-86.

Shoji, S., M. Nanzyo, and R.A. Dahlgren. 1993. Volcanic ash soils: Genesis, properties and utilization. Dev. Soil Sci. 21. Elsevier, Amsterdam, The Netherlands.

Singleton, P.L., M. McLeod, and H.J. Percival. 1989. Allophane and halloysite content and soil solution silicon in soils from rhyolitic volcanic material, New Zealand. Aust. J. Soil Res. 27:67-77.

Soil Survey Staff. 1996. Soil survey laboratory methods manual. Soil Survey Investigations Rep. 42. USDA-NRCS, Washington, DC.

Soil Survey Staff. 1998. Keys to soil taxonomy. 8th ed. USDA-NRCS, Washington, DC.

Stevens, K.F., and C.G. Vucetich. 1985. Weathering of Upper Quaternary tephras in New Zealand, 2. Clay minerals and their climatic interpretation. Chem. Geol. 53:237-247.

Tabatabai, M.A., and J.M. Bremner. 1991. Automated instruments for determination of total carbon, nitrogen, and sulfur in soils by combustion techniques. p. 261-286. In K.A. Smith (ed.) Soil analysis. Marcel Dekker, New York.

Takahashi, T., R. Dahlgren, and P. van Susteren. 1993. Clay mineralogy and chemistry of soils formed in volcanic materials in the xeric moisture regime of northern California. Geoderma 59:131-150.

Wada, K. 1980. Mineralogical characteristics of Andisols. p. 87-107. In B.K.G. Theng (ed.) Soils with variable charge. New Zealand Soc. Soil Sci., Offset Publications, Palmerston North, New Zealand.

Wada, K. 1987. Minerals formed and mineral formation from volcanic ash by weathering. Chem. Geol. 60:17-28.

Wada, K. 1989. Allophane and imogolite. p. 1051-1087. In J.B. Dixon and S.B. Weed (ed.) Minerals in soil environments. 2nd ed. SSSA Book Ser. 1. SSSA, Madison, WI.

Wada, K., and Y. Kakuto. 1985. Embryonic halloysites in Ecuadorian soils derived from volcanic ash. Soil Sci. Soc. Am. J. 49:1309-1318.

Yamada, I., and S. Shoji. 1983. Alteration of volcanic glass of recent Towada ash in different soil environments of northeastern Japan. Soil Sci. 135:316-321. 